\title{
Seven challenges in the multiscale modelling of multicellular tissues
}

Alexander G. Fletcher

School of Mathematics and Statistics, University of Sheffield, Sheffield S3 7RH, UK

Bateson Centre, University of Sheffield, Sheffield S10 2TN, UK

E-mail address: a.g.fletcher@sheffield.ac.uk

ORCID iD: https://orcid.org/0000-0003-0525-4336

James M. Osborne

School of Mathematics and Statistics, University of Melbourne, Parkville 3010, Victoria, Australia

Email address: imosborne@unimelb.edu.au

ORCID iD: https://orcid.org/0000-0002-5622-0104 


\section{ABSTRACT}

The growth and dynamics of multicellular tissues involve tightly regulated and coordinated morphogenetic cell behaviours, such as shape changes, movement, and division, which are governed by subcellular machinery and involve coupling through short- and long-range signals. A key challenge in the fields of developmental biology, tissue engineering and regeneration is to understand how relationships between scales produce emergent tissue-scale behaviours. Recent advances in molecular biology, live-imaging and ex vivo techniques have revolutionised our ability to study these processes experimentally. To fully leverage these techniques and obtain a more comprehensive understanding of the causal relationships underlying tissue dynamics, computational modelling approaches are increasingly spanning multiple spatial and temporal scales, and are coupling cell shape, growth, mechanics and signalling. Yet such models remain technically challenging: modelling at each scale requires different areas of technical skills, while integration across scales necessitates the solution to novel mathematical and computational problems. This review aims to summarise recent progress in multiscale modelling of multicellular tissues and to highlight ongoing challenges associated with the construction, implementation, interrogation and validation of such models. 


\section{INTRODUCTION}

How multicellular tissues self-organise and remodel is a fundamental question in developmental biology with profound implications for tissue engineering and regeneration (Sasai, 2013). Recent advances in molecular biology, live-imaging and ex vivo techniques have resulted in a wealth of detailed, but isolated, descriptions of these processes (St Johnston, 2015). To truly comprehend such complexity, we need computational models that can link observations in a quantitative, predictive, and reproducible way (Sharpe, 2017). However, before these goals can be achieved there are substantial methodological challenges to overcome. This review aims to highlight these challenges and how they might be overcome.

The growth and dynamics of multicellular tissues are multiscale in nature, involving tightly regulated and coordinated morphogenetic cell behaviours, such as shape changes, movement, and division, which are governed by subcellular machinery and involve coupling through shortand long-range signals (Figure 1). A key challenge is to understand how relationships between scales produce emergent tissue-scale self-organisation.

State-of-the-art experimental approaches are revealing emergent behaviour at different scales: super-resolution microscopy allows quantification of complex, dynamic feedback interactions and subcellular localisation (Liu \& Keller, 2016) underlying cell-level patterning; live-imaging and force-inference techniques uncover 'mesoscale' structures, comprising groups of cells, driving tissue morphogenesis (Blanchard et al., 2019); while ex vivo organoid cultures offer a window into the contribution and timing of different signals to tissue self-organisation (Simunovic \& Brivanlou, 2017). To fully leverage these techniques and obtain a more comprehensive understanding of the causal relationships underlying normal and abnormal tissue dynamics, computational modelling approaches are being developed that span multiple spatial and temporal scales, which couple cell shape, growth, mechanics and signalling.

A common approach to modelling the dynamics of multicellular tissues is to treat the tissue as a continuous material and apply classical techniques from continuum mechanics and thermodynamics. While continuum models continue to generate valuable mechanistic insights into multicellular tissue growth and dynamics, for example in characterizing the roles of mechanics in morphogenesis (Kaucka et al., 2017), they are ill-suited to investigate individual cell level heterogeneities. On the other hand, the ever decreasing cost of computing power has 
facilitated the accelerated development of discrete agent-based and multiscale modelling approaches, which more naturally allow investigation of the influence of intracellular and cellular level behaviours and heterogeneity in tissue-scale phenomena. Recent work on such models has facilitated successful interdisciplinary studies of emergent behaviours in developing tissues (Nissen et al., 2017); for a detailed review, see (Glen et al., 2019).

Despite their utility, agent-based and multiscale models of multicellular tissue remain technically challenging: modelling at each scale requires different areas of technical skills, while integration across scales necessitates the solution to novel mathematical and computational problems (Figure 1). In this review, we set out seven ongoing challenges in multicellular modelling, ordered roughly in the order in which they may be experienced by someone developing a new model of this form: from model construction and calibration; to numerical and software/hardware implementation; to analysis and validation; to issues associated with model re-use and comparing modelling assumptions and paradigms. For each challenge, we summarise recent progress in specific applications as well as efforts to tackle the general case, highlighting one key open research question that we believe requires attention in future computational research.

When commenting on applications of agent-based and multiscale modelling in the following, we focus primarily on animal developmental biology. This is a natural area in which to apply these modelling techniques, because of the availability of data across multiple spatial and temporal scales - from gene regulatory networks and signalling pathways to cellular interactions and larger-scale morphogen transport processes - and the feedbacks between scales that underlies many emergent processes (Sharpe, 2017). We refer the interested reader to several recent reviews on the use of these modelling approaches in plant biology (Bucksch et al., 2017), cancer biology (Metzcar et al., 2019), synthetic biology (Gorochowski et al., 2020), and tissue engineering (Montes-Olivas et al., 2019).

Our intention is to highlight progress and challenges in multiscale modelling in a way that is accessible to both practitioners (applied mathematicians, physicists and computer scientists) and stakeholders (experimental biologists and clinicians), and to help inform the agenda for future computational multiscale modelling efforts. 


\section{CHALLENGE 1: MODEL CONSTRUCTION}

In mathematical biology, modelling assumptions must be carefully tailored to the biological system of interest, the available data, and the scientific hypotheses. In the case of multiscale modelling, one must choose the level of complexity with which one describes subcellular processes, cellular interactions, and larger-scale processes such as morphogen transport. This leads to our first ongoing challenge: how to choose an appropriate model for a given problem.

A variety of multiscale modelling approaches have been developed for multicellular tissues (Fletcher et al., 2017). These approaches generally either discretise space into a regular (Lehotzky \& Zupanc, 2019) or irregular (Scianna \& Preziosi, 2016) lattice, or allow cells to move continuously in space (Drasdo \& Höhme, 2005). Such approaches also vary in their geometric description: cells may be treated as point-like particles or prescribed shapes such as ellipsoids (Xu et al., 2008); or cell shape may be more complex and dynamic, evolving according to a balance of forces (Farhadifar et al., 2007) or stochastic fluctuations (Graner \& Glazier, 1992). There can also be increased geometric complexity moving from two- and three-dimensional representations, such as a larger number of possible cell rearrangements (Okuda et al., 2015). Another distinction can be made based on the level of biophysical complexity used: considering cell surface mechanics only (Magno et al., 2015), or including subcellular heterogeneity such as the presence and mechanical role of the nucleus and other organelles (Jamali et al., 2010).

Several modelling challenges remain. For example, there is increasing evidence for the importance of subcellular spatial patterns of protein complexes for generation of cellular, and hence tissue, asymmetry (Strutt et al., 2011). This necessitates the development of more detailed models that include processes such as directed vesicular transport, trafficking and recycling, and cell shape changes. Other challenges include how to model cellular projections such as cytonemes and their role in cell-cell communication (Bressloff \& Kim, 2019; Rosenbauer et al., 2020) and the formation and functional roles of supracellular structures such as multicellular rosettes (Trichas et al., 2012) and supracellular actomyosin cables (Tetley et al., 2016), and more generally, mechanochemical feedbacks (Dasbiswas et al., 2018; Lan et al., 2015). We return to the issue of which models are well suited to address specific research questions, whether based on new or existing approaches, when discussing Challenge 7. 


\section{CHALLENGE 2: MODEL CALIBRATION}

Having arrived at a set of modelling assumptions, one is faced with the issue of how to choose appropriate parameter values and initial conditions. This leads to our second ongoing challenge: how to calibrate a multiscale model against data. Here we refer both to estimation of model parameters and their associated uncertainty, as well as calibration of initial and boundary conditions (for example, initial cell and tissue geometry). In an ideal world, we would parameterise any model and initialise our simulations using appropriate data at each level of the model. In practice, however, we must resort to using the understanding and data that are available to us (Pargett \& Umulis, 2013).

Initial conditions are specified based on our biological understanding. In some cases, this can be straightforward, for example when modelling tissue development from a single stem cell (Atwell et al., 2015). This ad hoc approach works well if there are initially few cells, or if the initial state is highly conserved. Another common approach is to evolve the system to a dynamic equilibrium that is not sensitive to the choice of initial condition, as in the case of many homeostatic tissues such as the skin (Li et al., 2013) or gut epithelium (van Leeuwen et al., 2009). For developing tissues undergoing morphogenesis, an alternative approach is to derive initial conditions such as cell packing directly based on microscopy images (lber et al., 2016) or via extracted summary statistics (Vicente-Munuera et al., 2019). Here, one should bear in mind variability in structures and it may be more important to capture essential patterns and conserved properties of tissue organization rather than use a specific example.

As well as establishing initial conditions such as cell and tissue geometry, one must arrive at values for model parameters. Typically, parameters cannot be measured directly but must be inferred from experimental data. Where quantitative datasets are available for parameter estimation, approximate Bayesian computation $(A B C)$ and its variants are increasingly used, since the likelihood of a multiscale model is typically neither analytically nor computationally tractable (Kursawe et al., 2018; Lambert et al., 2018). These approaches compare model outputs with experimental data and accept parameter values for which these are sufficiently close. The inference process is streamlined by the use of recent software tools such as pyABC (Klinger et al., 2018) and Pakman (Pak et al., 2020). These techniques can also be used to perform model selection when exploring two or more competing biological hypotheses in silico; we discuss the comparison of multiscale models in more detail when discussing Challenge 7. 
Local and global sensitivity analysis methods have also been developed to assess to which parameters a model is most sensitive, and to identify the effects of uncertainties in parameter values on its output (Boas et al., 2015).

Several challenges remain when specifying initial and boundary conditions and estimating parameters of multiscale models. In particular, more tailored and efficient parameter inference techniques are needed that rely less heavily on repeated simulations of computationally expensive models, for example by exploring parameter space more intelligently and by exploiting 'low-fidelity' or surrogate modelling (Prescott \& Baker, 2020). Where simulation outputs are intended to inform decision-making, greater use of uncertainty quantification techniques is needed, for which software tools are increasingly available (Richardson et al., 2020).

\section{CHALLENGE 3: NUMERICAL SOLUTION}

Due to their complexity, multiscale models of multicellular tissues are typically analytically intractable and must be solved numerically. This leads to our third challenge: how to solve multiscale models efficiently, stably and accurately. Their numerical solution remains challenging since the coupling of processes or sub-models across scales can make it harder to avoid numerical instabilities, and many such models include parameters of numerical approximation; for example, thresholds for changes in cell topology (Fletcher et al., 2013). Thus, we need to be aware of any impacts that numerical implementation choices may have on model predictions (Kursawe et al., 2017).

Typically, subcellular processes such as protein production, degradation and complex formation evolve on a faster timescale than cellular level processes such as division and movement, which in turn play out on a faster timescale than tissue-scale processes such as paracrine signalling. Therefore, based on quasi-steady state approximations or similar, multiscale models may be decomposed into subsystems that are solved numerically over discrete time steps during which other subsystems are held constant.

For deterministic systems, higher-order explicit numerical solvers are more computationally intensive but allow larger time steps to be used, while implicit solvers are algorithmically more 
complicated due to processes such as cell death and division changing the size of the dynamical system over each time step (Atwell, 2016). For some cell-based models, numerical methods must be carefully chosen to respect physical conservation laws (Cooper et al., 2017). Efficient numerical techniques have also been developed to simulate stochastic reactiondiffusion processes in spatially complex or evolving domains (Drawert et al., 2012). At the cellular level, algorithms have been developed to simulate the evolution of stochastic models such as the cellular Potts model efficiently while forbidding physically unrealistic phenomena such as cell fragmentation (Durand \& Guesnet, 2016). Several efforts have also been made to develop efficient solvers that couple cell-based models and tissue-scale processes such as nutrient transport (Ghaffarizadeh et al., 2016), morphogen signalling (Smith et al., 2012) and fluid flow (Grogan et al., 2017; Osborne \& Bernabeu, 2018), based on finite element, finite volume, finite difference, and lattice Boltzmann methods.

Looking ahead, ongoing challenges include the application of adaptive methods, which allow spatial or temporal discretizations to vary according, for example, to the amount of cell movement of proliferation in a domain. This also leads to the prospect of models that allow one to change the level of spatial complexity when and where needed, for example by using a cellbased description in regions where the bulk of growth occurs, and a continuum description elsewhere (Kim et al., 2007).

\section{CHALLENGE 4: SOFTWARE AND HARDWARE IMPLEMENTATION}

If every researcher implemented their own model from scratch we would have thousands of implementations of each model at a massive overall time cost. The solution to this is to develop and use computational tools to build and interrogate multicellular models, which leads to our fourth ongoing challenge: how to fully exploit modern software and hardware architectures. Multiple numerical libraries and software tools have been developed for building, running and interrogating multicellular models. These range from user-friendly GUls with limited functionality intended for use by biologists to more extensible software libraries, which require significant programming skills to utilise (Figure 2).

A key recent development has been the increased use of parallelisation and high performance computing (HPC). Two common use-cases for HPC, typically requiring different parallelisation 
approaches, are: to run a large number of simulations, each at reasonable computational cost (for example, when performing parameter sweeps); and to run a small number of simulations, each at significant computational cost (for example, when simulating the collective dynamics of tens or hundreds of thousands of cells).

The first use-case is 'trivially' parallelizable, in that multiple instances may be run independently on multiple processors in parallel. In contrast, the second use-case requires one to partition a simulated model into smaller components, for example through spatial decomposition, that are simulated in each processor. Parallel interfaces such as MPI (for distributed memory devices) and OpenMP (for shared memory devices) are then used to communicate transfer information between processors. Such algorithms have been developed, for example, for the cellular Potts model (Chen et al., 2007) and for off-lattice cell-centre models (Harvey et al., 2015). Further speedups may be obtained through the use of GPGUs, for example through using CUDA or OpenCL; again, published examples include implementations of the cellular Potts model (Tapia \& D'Souza, 2011) and off-lattice cell-centre models (Germann et al., 2019).

Several software and hardware challenges remain. When developing and refining software tools for multiscale modelling, one must carefully balance functionality and usability; such tools are unlikely to be taken up by non-specialists unless barriers are further lowered, for example through the greater use of GUls and APIs. Modern computing resources, such as cloud computing, should also be more fully exploited; a laudable example of work towards this is xml2jupyter (Heiland et al., 2019). Finally, there remains remarkably little in the way of crossvalidation of different model implementations; we return to this point when discussing Challenges 6 and 7 .

\section{CHALLENGE 5: MODEL VALIDATION}

A multiscale model may be used to leverage existing data and obtain a more comprehensive understanding of the causal relationships underlying tissue self-organisation and remodelling. If a model reproduces key observations in a robust and reproducible way, then a natural next step is to use the model to generate experimentally testable predictions for what we should observe under different circumstances, for example when the system undergoes a genetic or mechanical perturbation. By testing these predictions, we can seek to validate the underlying model assumptions. This leads to our fifth ongoing challenge: how to validate multiscale models 
against data? This strongly relates to the issue of parameter inference and model selection discussed in Challenge 2, but is typically encountered later in the modelling process. In each case, we typically have access only to partial information about the system at each spatial or temporal scale, and so we may need to resort to validating separate model components individually (Viceconti et al., 2020).

Testing model predictions, like estimating model parameters (see Challenge 2), often involves using various different types and sources of experimental data. Subcellular processes, such as protein dynamics, may be compared - at least semi-quantitatively - against experiments on isolated cells or on in vitro cell cultures (Tan et al., 2012), with due care for possible discrepancies with the in vivo setting. At the cellular and tissue level, rheology experiments, laser ablations, and non-invasive force inference techniques can all be used to interrogate how tissues respond to applied stresses (Sugimura et al., 2016) and compare these measurements with model predictions. Cell tracking can also be used to match cell velocities and other summary statistics. In this context, recent work demonstrating the pitfalls of inferring cell-level heterogeneity from such data (Schumacher et al., 2017) has implications for inferring parameters from the 'wrong' model; we return to the issue of comparing models and modelling assumptions when discussing Challenge 7.

A complementary approach to validating multiscale models is to apply mathematical or computational techniques to perform system model reduction, for example through the use of asymptotic or equation-free methods. Here, the intention is to arrive at a coarse-grained model that may be more amenable to efficient simulation or qualitative mathematical analysis; such approaches have been applied, to lattice-based models (Johnston et al., 2012) and cellular Potts models (Alber et al., 2007), as well as off-lattice cell-centre models (Murray et al., 2012) and vertex models (Fozard et al., 2010). Further work is needed in this area, as well as advances in our statistical understanding of how best to use information at different scales, the extent to which we can fully validate a given model, and which experiments will yield the greatest information for model calibration and validation.

\section{CHALLENGE 6: DATA / CODE STANDARDS AND BENCHMARKS}


There are myriad simulation tools, as mentioned above. This is unlike fields like molecular dynamics where one or two libraries are used by everyone or 'subcellular' systems biology where the modelling formalism is shared across the field. This leads to our sixth ongoing challenge: how to assess robustness of simulation results to software and hardware implementation.

There are ongoing discussions in the wider field of computational biology regarding the need for model replicability and reproducibility (Grüning et al., 2018; Lewis et al., 2016). In the case of multiscale modelling of multicellular tissues, model construction, exchange and re-use remain hampered by the lack of easily shareable model definitions, for example in the form of a fully declarative model definition language. SBML and CellML have largely solved this problem in the context of reaction kinetics, and have associated APIs and model repositories, but have limited functionality for spatial models; some initiatives are ongoing in this area, such as the MultiCellML project. Many multicellular tools have support for SBML specification of subcellular processes, for example CompuCell3D (Swat et al., 2012), Chaste (Romijn et al., 2020) and Morpheus (Starruß et al., 2014). Another aspect of this is separating out the definition of the biological process from the definition of any in silico experiments or perturbations, allowing easier comparison of model behaviour with experimental data. Relatively little work has been done on this (J. Cooper \& Osborne, 2013) compared to more mature areas of systems biology such as cardiac electrophysiology (Cooper et al., 2016).

Several challenges remain in this area. In particular, we must work out how to improve standards, reproducibility and interoperability among new and existing software tools (Macklin, 2019). Here, there may be lessons to be learned from other domains such as astrophysics, weather/climate modelling, and molecular dynamics.

\section{CHALLENGE 7: COMPARING MODELLING ASSUMPTIONS AND APPROACHES}

As outlined above, there are many ways to model the dynamics of individual cells and their interactions within tissues, to solve a model numerically, and to implement this in the computer. This leads to our final ongoing challenge: how to assess robustness of model behaviour to choice of implementation. This is of particular relevance to biomedical applications, if predictions are to inform clinical practice. 
It can be difficult to disentangle differences in model behaviour arising from algorithmic, software, and hardware implementation. However, some progress has been made on aspects of this issue. Simulation studies have been used to compare different mechanical assumptions and constitutive equations for particular classes of cell-based models, such as cell-centre models (Mathias et al., 2020; Pathmanathan et al., 2009) and vertex models (Fletcher et al., 2013). Efforts have also been made to compare and contrast five competing cell-based model paradigms, including lattice-based and off-lattice models, in a consistent computational framework (Osborne et al., 2017), to help elucidate where one may expect to see qualitative differences between model behaviours. Four exemplar simulations were chosen to compare the models, which represent facets of development: adhesion; proliferation, death and differentiation; short-range signalling; and long-range signalling. At a tissue level, each of the modelling paradigms investigated was able to implement these processes and, outside a few edge cases where artifacts were introduced, the tissue level behaviour was qualitatively the same. A natural extension of this work is to compare models across different software implementations, such as those discussed in Challenge 6. This is feasible in principle, though one needs to ensure the same model definition and numerical method of solution (to within some tolerance), which is difficult in the absence of model standards discussed above.

Recent work has begun comparing the effect of both cellular and subcellular models especially in the field of intestinal crypt development (Dunn et al., 2013) compare how multiple cell level models of the crypt behave under artificial radiation and compare this to decades-old experimental results, adding new insight into the mechanism for cell migration in the absence of mitosis; while elsewhere a framework to couple SBML subcellular models with multicellular crypt simulations has been used to compare different approaches to modelling the Wnt pathway (Romijn et al., 2020).

In addition, the challenge of defining a suitable set of benchmark biological problems with which to simulate the model remains. Examples for comparison need to be chosen to identify similarities and differences between model paradigms. Despite recent workshops and collaborative modelling endeavours we are still only starting the journey of simulations specification and model comparison and both of these form ongoing challenges. 


\section{CONCLUSION}

In this paper we have presented seven open challenges associated with multiscale modelling of multicellular tissue dynamics. These challenges range from the mathematical (how to couple processes across multiple scales) and statistical (how to infer multiscale model parameters from partial information on processes at each scale) to the computational (how to unambiguously define such models in a shareable format) and even societal (how to exploit synergies among competing software tools). In our view, overcoming these challenges will require a significant community-driven effort in order to move such modelling approaches to a point where they can be a standard tool for stem cell and developmental biologists and tissue engineers alongside experimental approaches. Addressing these challenges, particularly those associated with quantifying uncertainty in model predictions, is even more important if such approaches are to become a greater part of clinical research and decision-making, as is starting to be seen for example in mathematical oncology (Rockne et al., 2019).

\section{FUNDING INFORMATION}

AGF acknowledges the support of the UK Biotechnology and Biological Sciences Research Council (BBSRC, grant BB/R016925/1) and the University of Sheffield (Vice-Chancellor's Fellowship).

\section{ACKNOWLEDGMENTS}

AGF thanks the attendees of the workshop "Tissue Mechanics and Genetics 2018" for their discussion on numerical simulations of tissue morphogenesis, on which this review is inspired. The authors also thank the attendees at the 2018 MATRIX workshop on "Virtual tissues: progress and challenges in Multicellular Systems Biology" for their discussion on model comparison specification which have inspired the challenges discussed here. The authors are grateful to Ian Groves, Jochen Kursawe, and Mari Herigstad for insightful comments on this manuscript. 


\section{REFERENCES}

Atwell, K. (2016). Investigating the interplay between cellular mechanics and decision-making in the C. elegans germ line [PhD Thesis]. University of Oxford.

Atwell, K., Qin, Z., Gavaghan, D., Kugler, H., Hubbard, E. J. A., \& Osborne, J. M. (2015). Mechano-logical model of $\mathrm{C}$. elegans germ line suggests feedback on the cell cycle. Development, 142(22), 3902-3911. https://doi.org/10.1242/dev.126359

Blanchard, G. B., Fletcher, A. G., \& Schumacher, L. J. (2019). The devil is in the mesoscale: Mechanical and behavioural heterogeneity in collective cell movement. Seminars in Cell \& Developmental Biology, 93, 46-54. https://doi.org/10.1016/j.semcdb.2018.06.003

Boas, S. E. M., Navarro Jimenez, M. I., Merks, R. M. H., \& Blom, J. G. (2015). A global sensitivity analysis approach for morphogenesis models. BMC Systems Biology, 9(1), 85. https://doi.org/10.1186/s12918-015-0222-7

Bravo, R. R., Baratchart, E., West, J., Schenck, R. O., Miller, A. K., Gallaher, J., Gatenbee, C. D., Basanta, D., Robertson-Tessi, M., \& Anderson, A. R. A. (2020). Hybrid Automata Library: A flexible platform for hybrid modeling with real-time visualization. PLOS Computational Biology, 16(3), e1007635. https://doi.org/10.1371/journal.pcbi.1007635

Bressloff, P. C., \& Kim, H. (2019). Search-and-capture model of cytoneme-mediated morphogen gradient formation. Physical Review E, 99(5), 052401. https://doi.org/10.1103/PhysRevE.99.052401

Bucksch, A., Atta-Boateng, A., Azihou, A. F., Battogtokh, D., Baumgartner, A., Binder, B. M., Braybrook, S. A., Chang, C., Coneva, V., DeWitt, T. J., Fletcher, A. G., Gehan, M. A., Diaz-Martinez, D. H., Hong, L., lyer-Pascuzzi, A. S., Klein, L. L., Leiboff, S., Li, M., Lynch, J. P., ... Chitwood, D. H. (2017). Morphological plant modeling: Unleashing geometric and topological potential within the plant sciences. Frontiers in Plant Science, 8, 900. https://doi.org/10.3389/fpls.2017.00900 
Cooper, F., Baker, R., Bernabeu, M., Bordas, R., Bowler, L., Bueno-Orovio, A., Byrne, H., Carapella, V., Cardone-Noott, L., Cooper, J., Dutta, S., Evans, B., Fletcher, A., Grogan, J., Guo, W., Harvey, D., Hendrix, M., Kay, D., Kursawe, J., ... Gavaghan, D. (2020). Chaste: Cancer, Heart and Soft Tissue Environment. Journal of Open Source Software, 5(47), 1848. https://doi.org/10.21105/joss. 01848

Cooper, F. R., Baker, R. E., \& Fletcher, A. G. (2017). Numerical analysis of the immersed boundary method for cell-based simulation. SIAM Journal on Scientific Computing, 39(5), B943-B967. https://doi.org/10.1137/16M1092246

Cooper, J., Scharm, M., \& Mirams, G. R. (2016). The Cardiac Electrophysiology Web Lab. Biophysical Journal, 110(2), 292-300. https://doi.org/10.1016/j.bpj.2015.12.012

Dasbiswas, K., Hannezo, E., \& Gov, N. S. (2018). Theory of epithelial cell shape transitions induced by mechanoactive chemical gradients. Biophysical Journal, 114(4), 968-977. https://doi.org/10.1016/j.bpj.2017.12.022

Delile, J., Herrmann, M., Peyriéras, N., \& Doursat, R. (2017). A cell-based computational model of early embryogenesis coupling mechanical behaviour and gene regulation. Nature Communications, 8(1), 13929. https://doi.org/10.1038/ncomms13929

Drasdo, D., \& Höhme, S. (2005). A single-cell-based model of tumor growth in vitro: Monolayers and spheroids. Physical Biology, 2(3), 133-147. https://doi.org/10.1088/1478$3975 / 2 / 3 / 001$

Drawert, B., Engblom, S., \& Hellander, A. (2012). URDME: A modular framework for stochastic simulation of reaction-transport processes in complex geometries. BMC Systems Biology, 6(1), 76. https://doi.org/10.1186/1752-0509-6-76

Dunn, S.-J., Näthke, I. S., \& Osborne, J. M. (2013). Computational models reveal a passive mechanism for cell migration in the crypt. PLOS ONE, 8(11), e80516. https://doi.org/10.1371/journal.pone.0080516

Durand, M., \& Guesnet, E. (2016). An efficient Cellular Potts Model algorithm that forbids cell 
fragmentation. Computer Physics Communications, 208, 54-63. https://doi.org/10.1016/j.cpc.2016.07.030

Farhadifar, R., Röper, J.-C., Aigouy, B., Eaton, S., \& Jülicher, F. (2007). The influence of cell mechanics, cell-cell interactions, and proliferation on epithelial packing. Current Biology, 17(24), 2095-2104. https://doi.org/10.1016/j.cub.2007.11.049

Fletcher, A. G., Cooper, F., \& Baker, R. E. (2017). Mechanocellular models of epithelial morphogenesis. Philosophical Transactions of the Royal Society B: Biological Sciences, 372(1720), 20150519. https://doi.org/10.1098/rstb.2015.0519

Fletcher, A. G., Osborne, J. M., Maini, P. K., \& Gavaghan, D. J. (2013). Implementing vertex dynamics models of cell populations in biology within a consistent computational framework. Progress in Biophysics and Molecular Biology, 113(2), 299-326. https://doi.org/10.1016/j.pbiomolbio.2013.09.003

Fozard, J. A., Byrne, H. M., Jensen, O. E., \& King, J. R. (2010). Continuum approximations of individual-based models for epithelial monolayers. Mathematical Medicine and Biology, 27(1), 39-74. https://doi.org/10.1093/imammb/dqp015

Germann, P., Marin-Riera, M., \& Sharpe, J. (2019). ya||a: GPU-powered spheroid models for mesenchyme and epithelium. Cell Systems, 8(3), 261-266.e3. https://doi.org/10.1016/j.cels.2019.02.007

Ghaffarizadeh, A., Friedman, S. H., \& Macklin, P. (2016). BioFVM: An efficient, parallelized diffusive transport solver for 3-D biological simulations. Bioinformatics, 32(8), 12561258. https://doi.org/10.1093/bioinformatics/btv730

Ghaffarizadeh, A., Heiland, R., Friedman, S. H., Mumenthaler, S. M., \& Macklin, P. (2018). PhysiCell: An open source physics-based cell simulator for 3-D multicellular systems. PLOS Computational Biology, 14(2), e1005991. https://doi.org/10.1371/journal.pcbi.1005991

Glen, C. M., Kemp, M. L., \& Voit, E. O. (2019). Agent-based modeling of morphogenetic 
systems: Advantages and challenges. PLOS Computational Biology, 15(3), e1006577. https://doi.org/10.1371/journal.pcbi.1006577

Gorochowski, T. E., Hauert, S., Kreft, J., Marucci, L., Stillman, N. R., Tang, T. D., Bandiera, L., Bartoli, V., Dixon, D. O. R., Fedorec, A. J. H., Fellermann, H., Fletcher, A. G., Foster, T., Giuggiolo, L., Matyjazkiewicz, A., McCormick, S., Montes Olivas, S., Naylor, J., Rubio Deniss, A., \& Ward, D. (2020). Towards engineering biosystems with emergent collective functions. In press.

Graner, F., \& Glazier, J. A. (1992). Simulation of biological cell sorting using a two-dimensional extended Potts model. Physical Review Letters, 69(13), 2013-2016. https://doi.org/10.1103/PhysRevLett.69.2013

Grogan, J. A., Connor, A. J., Markelc, B., Muschel, R. J., Maini, P. K., Byrne, H. M., \& PittFrancis, J. M. (2017). Microvessel Chaste: An open library for spatial modeling of vascularized tissues. Biophysical Journal, 112(9), 1767-1772. https://doi.org/10.1016/j.bpj.2017.03.036

Grüning, B., Chilton, J., Köster, J., Dale, R., Soranzo, N., van den Beek, M., Goecks, J., Backofen, R., Nekrutenko, A., \& Taylor, J. (2018). Practical computational reproducibility in the life sciences. Cell Systems, 6(6), 631-635. https://doi.org/10.1016/j.cels.2018.03.014

Heiland, R., Mishler, D., Zhang, T., Bower, E., \& Macklin, P. (2019). xml2jupyter: Mapping parameters between XML and Jupyter widgets. Journal of Open Source Software, 4(39), 1408. https://doi.org/10.21105/joss.01408

Hoehme, S., \& Drasdo, D. (2010). A cell-based simulation software for multi-cellular systems. Bioinformatics, 26(20), 2641-2642. https://doi.org/10.1093/bioinformatics/btq437 Iber, D., Karimaddini, Z., \& Ünal, E. (2016). Image-based modelling of organogenesis. Briefings in Bioinformatics, 17(4), 616-627. https://doi.org/10.1093/bib/bbv093

Jamali, Y., Azimi, M., \& Mofrad, M. R. K. (2010). A sub-cellular viscoelastic model for cell 
population mechanics. PLOS ONE, 5(8), e12097.

https://doi.org/10.1371/journal.pone.0012097

Johnston, S. T., Simpson, M. J., \& Baker, R. E. (2012). Mean-field descriptions of collective migration with strong adhesion. Physical Review E, 85(5), 051922. https://doi.org/10.1103/PhysRevE.85.051922

Kang, S., Kahan, S., McDermott, J., Flann, N., \& Shmulevich, I. (2014). Biocellion: Accelerating computer simulation of multicellular biological system models. Bioinformatics, 30(21), 3101-3108. https://doi.org/10.1093/bioinformatics/btu498

Kaucka, M., Zikmund, T., Tesarova, M., Gyllborg, D., Hellander, A., Jaros, J., Kaiser, J., Petersen, J., Szarowska, B., Newton, P. T., Dyachuk, V., Li, L., Qian, H., Johansson, A.S., Mishina, Y., Currie, J. D., Tanaka, E. M., Erickson, A., Dudley, A., ... Adameyko, I. (2017). Oriented clonal cell dynamics enables accurate growth and shaping of vertebrate cartilage. ELife, 6, e25902. https://doi.org/10.7554/eLife.25902

Klinger, E., Rickert, D., \& Hasenauer, J. (2018). pyABC: Distributed, likelihood-free inference. Bioinformatics, 34(20), 3591-3593. https://doi.org/10.1093/bioinformatics/bty361

Kursawe, J., Baker, R. E., \& Fletcher, A. G. (2017). Impact of implementation choices on quantitative predictions of cell-based computational models. Journal of Computational Physics, 345, 752-767. https://doi.org/10.1016/j.jcp.2017.05.048

Kursawe, J., Baker, R. E., \& Fletcher, A. G. (2018). Approximate Bayesian computation reveals the importance of repeated measurements for parameterising cell-based models of growing tissues. Journal of Theoretical Biology, 443, 66-81. https://doi.org/10.1016/j.jtbi.2018.01.020

Lambert, B., MacLean, A. L., Fletcher, A. G., Combes, A. N., Little, M. H., \& Byrne, H. M. (2018). Bayesian inference of agent-based models: A tool for studying kidney branching morphogenesis. Journal of Mathematical Biology, 76(7), 1673-1697. https://doi.org/10.1007/s00285-018-1208-z 
Lan, H., Wang, Q., Fernandez-Gonzalez, R., \& Feng, J. J. (2015). A biomechanical model for cell polarization and intercalation during Drosophila germband extension. Physical Biology, 12(5), 056011. https://doi.org/10.1088/1478-3975/12/5/056011

Lehotzky, D., \& Zupanc, G. K. H. (2019). Cellular automata modeling of stem-cell-driven development of tissue in the nervous system. Developmental Neurobiology, dneu.22686. https://doi.org/10.1002/dneu.22686

Lewis, J., Breeze, C. E., Charlesworth, J., Maclaren, O. J., \& Cooper, J. (2016). Where next for the reproducibility agenda in computational biology? BMC Systems Biology, 10(1), 52. https://doi.org/10.1186/s12918-016-0288-x

Li, X., Upadhyay, A. K., Bullock, A. J., Dicolandrea, T., Xu, J., Binder, R. L., Robinson, M. K., Finlay, D. R., Mills, K. J., Bascom, C. C., Kelling, C. K., Isfort, R. J., Haycock, J. W., MacNeil, S., \& Smallwood, R. H. (2013). Skin stem cell hypotheses and long term clone survival_Explored using agent-based modelling. Scientific Reports, 3(1), 1904. https://doi.org/10.1038/srep01904

Liu, Z., \& Keller, P. J. (2016). Emerging Imaging and Genomic Tools for Developmental Systems Biology. Developmental Cell, 36(6), 597-610. https://doi.org/10.1016/j.devcel.2016.02.016

Macklin, P. (2019). Key challenges facing data-driven multicellular systems biology. GigaScience, 8(10), giz127. https://doi.org/10.1093/gigascience/giz127

Magno, R., Grieneisen, V. A., \& Marée, A. F. (2015). The biophysical nature of cells: Potential cell behaviours revealed by analytical and computational studies of cell surface mechanics. BMC Biophysics, 8(1), 8. https://doi.org/10.1186/s13628-015-0022-x

Marin-Riera, M., Brun-Usan, M., Zimm, R., Välikangas, T., \& Salazar-Ciudad, I. (2015). Computational modeling of development by epithelia, mesenchyme and their interactions: A unified model. Bioinformatics, 32(2), 219-225. https://doi.org/10.1093/bioinformatics/btv527 
Mathias, S., Coulier, A., Bouchnita, A., \& Hellander, A. (2020). Impact of force function formulations on the numerical simulation of centre-based models [Preprint]. Biophysics. https://doi.org/10.1101/2020.03.16.993246

Metzcar, J., Wang, Y., Heiland, R., \& Macklin, P. (2019). A review of cell-based computational modeling in cancer biology. JCO Clinical Cancer Informatics, 3, 1-13. https://doi.org/10.1200/CCI.18.00069

Montes-Olivas, S., Marucci, L., \& Homer, M. (2019). Mathematical models of organoid cultures. Frontiers in Genetics, 10, 873. https://doi.org/10.3389/fgene.2019.00873

Murray, P. J., Edwards, C. M., Tindall, M. J., \& Maini, P. K. (2012). Classifying general nonlinear force laws in cell-based models via the continuum limit. Physical Review E, 85(2), 021921. https://doi.org/10.1103/PhysRevE.85.021921

Nissen, S. B., Perera, M., Gonzalez, J. M., Morgani, S. M., Jensen, M. H., Sneppen, K., Brickman, J. M., \& Trusina, A. (2017). Four simple rules that are sufficient to generate the mammalian blastocyst. PLOS Biology, 15(7), e2000737. https://doi.org/10.1371/journal.pbio.2000737

Osborne, J. M., \& Bernabeu, M. O. (2018). A fully discrete open source framework for the simulation of vascular remodelling. 2018 40th Annual International Conference of the IEEE Engineering in Medicine and Biology Society (EMBC), 4552-4555. https://doi.org/10.1109/EMBC.2018.8513223

Osborne, J. M., Fletcher, A. G., Pitt-Francis, J. M., Maini, P. K., \& Gavaghan, D. J. (2017). Comparing individual-based approaches to modelling the self-organization of multicellular tissues. PLOS Computational Biology, 13(2), e1005387. https://doi.org/10.1371/journal.pcbi.1005387

Pak, T., Baker, R., \& Pitt-Francis, J. (2020). Pakman: A modular, efficient and portable tool for approximate Bayesian inference. Journal of Open Source Software, 5(47), 1716. https://doi.org/10.21105/joss.01716 
Pargett, M., \& Umulis, D. M. (2013). Quantitative model analysis with diverse biological data: Applications in developmental pattern formation. Methods, 62(1), 56-67. https://doi.org/10.1016/j.ymeth.2013.03.024

Pathmanathan, P., Cooper, J., Fletcher, A., Mirams, G., Murray, P., Osborne, J., Pitt-Francis, J., Walter, A., \& Chapman, S. J. (2009). A computational study of discrete mechanical tissue models. Physical Biology, 6(3), 036001. https://doi.org/10.1088/1478$3975 / 6 / 3 / 036001$

Prescott, T. P., \& Baker, R. E. (2020). Multifidelity approximate Bayesian computation. SIAM/ASA Journal on Uncertainty Quantification, 8(1), 114-138. https://doi.org/10.1137/18M1229742

Richardson, R. A., Wright, D. W., Edeling, W., Jancauskas, V., Lakhlili, J., \& Coveney, P. V. (2020). EasyVVUQ: A library for verification, validation and uncertainty quantification in high performance computing. Journal of Open Research Software, 8, 11. https://doi.org/10.5334/jors.303

Rockne, R. C., Hawkins-Daarud, A., Swanson, K. R., Sluka, J. P., Glazier, J. A., Macklin, P., Hormuth, D. A., Jarrett, A. M., Lima, E. A. B. F., Tinsley Oden, J., Biros, G., Yankeelov, T. E., Curtius, K., Al Bakir, I., Wodarz, D., Komarova, N., Aparicio, L., Bordyuh, M., Rabadan, R., ... Scott, J. G. (2019). The 2019 mathematical oncology roadmap. Physical Biology, 16(4), 041005. https://doi.org/10.1088/1478-3975/ab1a09

Romijn, L. B., Almet, A. A., Tan, C. W., \& Osborne, J. M. (2020). Modelling the effect of subcellular mutations on the migration of cells in the colorectal crypt. BMC Bioinformatics, 21(1), 95. https://doi.org/10.1186/s12859-020-3391-3

Rosenbauer, J., Zhang, C., Mattes, B., Reinartz, I., Wedgwood, K., Schindler, S., Sinner, C., Scholpp, S., \& Schug, A. (2020). Modeling of Wnt-mediated tissue patterning in vertebrate embryogenesis. PLOS Computational Biology, 16(6), e1007417. https://doi.org/10.1371/journal.pcbi.1007417 
Sasai, Y. (2013). Cytosystems dynamics in self-organization of tissue architecture. Nature, 493(7432), 318-326. https://doi.org/10.1038/nature11859

Schumacher, L. J., Maini, P. K., \& Baker, R. E. (2017). Semblance of Heterogeneity in Collective Cell Migration. Cell Systems, 5(2), 119-127.e1. https://doi.org/10.1016/j.cels.2017.06.006

Scianna, M., \& Preziosi, L. (2016). A node-based version of the cellular Potts model. Computers in Biology and Medicine, 76, 94-112. https://doi.org/10.1016/j.compbiomed.2016.06.027

Sharpe, J. (2017). Computer modeling in developmental biology: Growing today, essential tomorrow. Development, 144(23), 4214-4225. https://doi.org/10.1242/dev.151274

Simunovic, M., \& Brivanlou, A. H. (2017). Embryoids, organoids and gastruloids: New approaches to understanding embryogenesis. Development, 144(6), 976-985. https://doi.org/10.1242/dev.143529

Smith, A. M., Baker, R. E., Kay, D., \& Maini, P. K. (2012). Incorporating chemical signalling factors into cell-based models of growing epithelial tissues. Journal of Mathematical Biology, 65(3), 441-463. https://doi.org/10.1007/s00285-011-0464-y

St Johnston, D. (2015). The renaissance of developmental biology. PLOS Biology, 13(5), e1002149. https://doi.org/10.1371/journal.pbio.1002149

Starruß, J., de Back, W., Brusch, L., \& Deutsch, A. (2014). Morpheus: A user-friendly modeling environment for multiscale and multicellular systems biology. Bioinformatics, 30(9), 1331-1332. https://doi.org/10.1093/bioinformatics/btt772

Strutt, H., Warrington, S. J., \& Strutt, D. (2011). Dynamics of core planar polarity protein turnover and stable assembly into discrete membrane subdomains. Developmental Cell, 20(4), 511-525. https://doi.org/10.1016/j.devcel.2011.03.018

Sugimura, K., Lenne, P.-F., \& Graner, F. (2016). Measuring forces and stresses in situ in living tissues. Development, 143(2), 186-196. https://doi.org/10.1242/dev.119776

Sutterlin, T., Kolb, C., Dickhaus, H., Jager, D., \& Grabe, N. (2013). Bridging the scales: 
Semantic integration of quantitative SBML in graphical multi-cellular models and simulations with EPISIM and COPASI. Bioinformatics, 29(2), 223-229. https://doi.org/10.1093/bioinformatics/bts659

Swat, M. H., Thomas, G. L., Belmonte, J. M., Shirinifard, A., Hmeljak, D., \& Glazier, J. A. (2012). Multi-scale modeling of tissues using CompuCell3D. In Methods in Cell Biology (Vol. 110, pp. 325-366). Elsevier. https://doi.org/10.1016/B978-0-12-388403-9.00013-8

Tan, C. W., Gardiner, B. S., Hirokawa, Y., Layton, M. J., Smith, D. W., \& Burgess, A. W. (2012). Wnt signalling pathway parameters for mammalian cells. PLoS ONE, 7(2), e31882. https://doi.org/10.1371/journal.pone.0031882

Tanaka, S., Sichau, D., \& Iber, D. (2015). LBIBCell: A cell-based simulation environment for morphogenetic problems. Bioinformatics, 31(14), 2340-2347. https://doi.org/10.1093/bioinformatics/btv147

Tetley, R. J., Blanchard, G. B., Fletcher, A. G., Adams, R. J., \& Sanson, B. (2016). Unipolar distributions of junctional Myosin II identify cell stripe boundaries that drive cell intercalation throughout Drosophila axis extension. ELife, 5. https://doi.org/10.7554/eLife.12094

Trichas, G., Smith, A. M., White, N., Wilkins, V., Watanabe, T., Moore, A., Joyce, B., Sugnaseelan, J., Rodriguez, T. A., Kay, D., Baker, R. E., Maini, P. K., \& Srinivas, S. (2012). Multi-cellular rosettes in the mouse visceral endoderm facilitate the ordered migration of anterior visceral endoderm cells. PLoS Biology, 10(2), e1001256. https://doi.org/10.1371/journal.pbio.1001256

van Leeuwen, I. M. M., Mirams, G. R., Walter, A., Fletcher, A., Murray, P., Osborne, J., Varma, S., Young, S. J., Cooper, J., Doyle, B., Pitt-Francis, J., Momtahan, L., Pathmanathan, P., Whiteley, J. P., Chapman, S. J., Gavaghan, D. J., Jensen, O. E., King, J. R., Maini, P. K., ... Byrne, H. M. (2009). An integrative computational model for intestinal tissue renewal. Cell Proliferation, 42(5), 617-636. https://doi.org/10.1111/j.1365- 
2184.2009.00627.x

Viceconti, M., Juarez, M. A., Curreli, C., Pennisi, M., Russo, G., \& Pappalardo, F. (2020). Credibility of in silico trial technologies-A theoretical framing. IEEE Journal of Biomedical and Health Informatics, 24(1), 4-13. https://doi.org/10.1109/JBHI.2019.2949888

Vicente-Munuera, P., Gómez-Gálvez, P., Tetley, R. J., Forja, C., Tagua, A., Letrán, M., Tozluoglu, M., Mao, Y., \& Escudero, L. M. (2019). EpiGraph: An open-source platform to quantify epithelial organization. Bioinformatics, btz683. https://doi.org/10.1093/bioinformatics/btz683

Wolff, H. B., Davidson, L. A., \& Merks, R. M. H. (2019). Adapting a plant tissue model to animal development: Introducing cell sliding into VirtualLeaf. Bulletin of Mathematical Biology, 81(8), 3322-3341. https://doi.org/10.1007/s11538-019-00599-9

Xu, Z., Chen, N., Kamocka, M. M., Rosen, E. D., \& Alber, M. (2008). A multiscale model of thrombus development. Journal of The Royal Society Interface, 5(24), 705-722. https://doi.org/10.1098/rsif.2007.1202 


\section{FIGURE 1}

Scale

Biological processes

Protein interactions, localisation, turnover, signal transduction

Cell shape changes, polarisation, movement, division, apoptosis

Actomyosin cables, multicellular rosettes

Convergent extension, size control, morphogen gradients

\section{Modelling approaches}

Differential equations, master equations

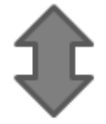

Cellular Potts models, vertex models, finite element models

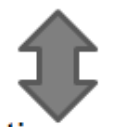

Hybrid / effective continuum models

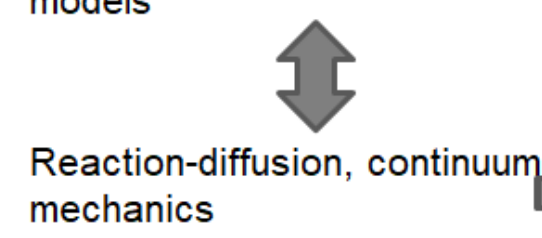

Modelling multiscale mechanisms of tissue self-organisation and remodelling. Hierarchy of spatial scales with associated biological examples and example modelling approaches. Light arrows show interdependence between scales. Dark arrows show where new mathematical models are required that bridge scales. 


\section{FIGURE 2}

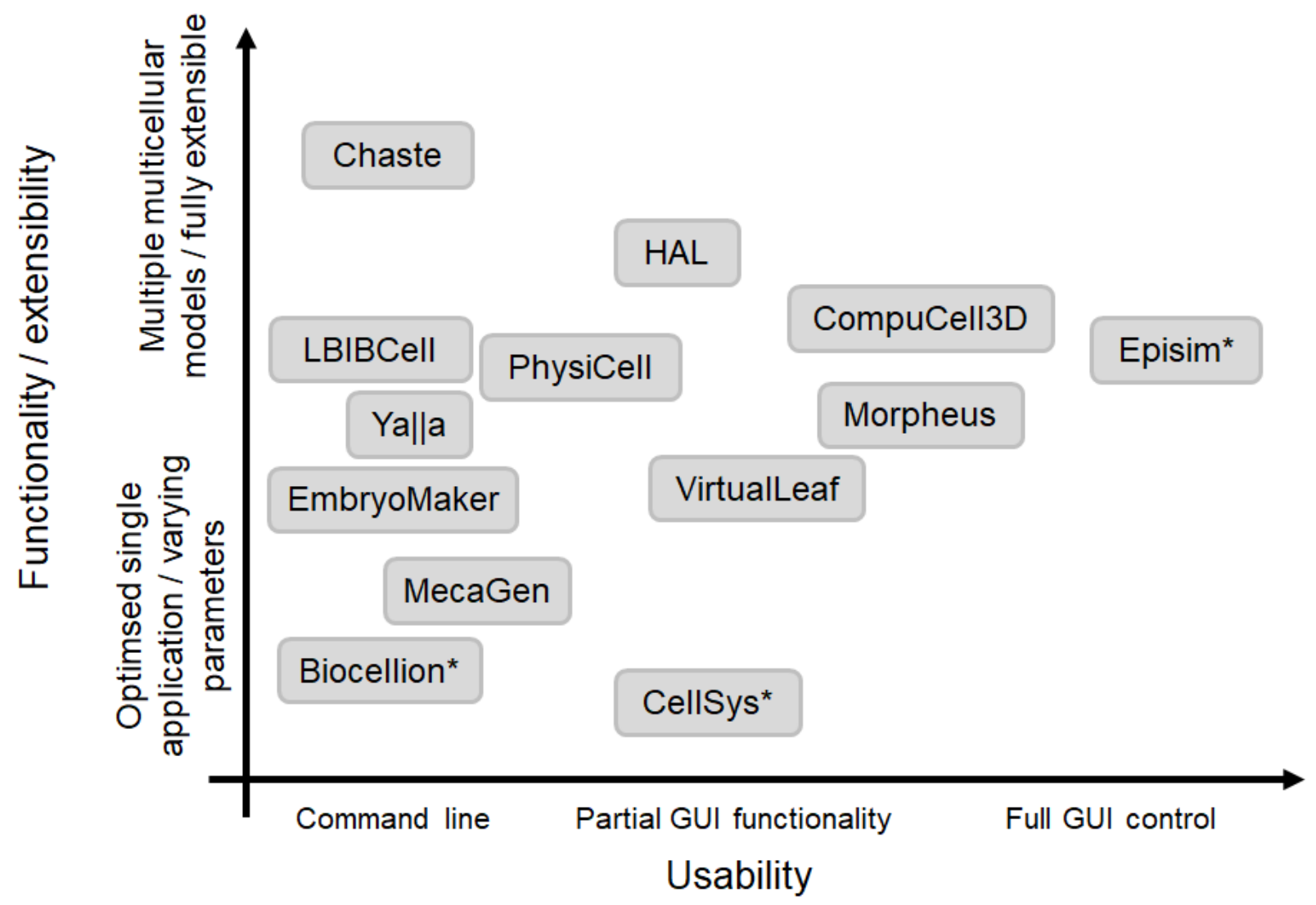

Summary of existing software tools for multiscale modelling of multicellular tissues based roughly on their usability and functionality/extensibility. We define usability by the level of graphical user interface (GUI) support for model construction, visualisation and analysis; we do not account for ease of use in any greater detail, since this depends strongly on user background and experience. We define functionality/extensibility in terms of the range of possible multicellular modelling assumptions that may be implemented; we do not account for other factors such as use of markup languages to import submodels, nor level of HPC or GPGPU support, since this can change rapidly. Asterisks denote tools that are not open source. References for these tools are as follows: Biocellion (Kang et al., 2014); CellSys (Hoehme \& Drasdo, 2010); Chaste (Cooper et al., 2020); CompuCell3D (Swat et al., 2012); EmbryoMaker (Marin-Riera et al., 2015); Episim (Sutterlin et al., 2013); HAL (Bravo et al., 2020); LBIBCell (Tanaka et al., 2015); MecaGen (Delile et al., 2017); Morpheus (Starruß et al., 2014), PhysiCell (Ghaffarizadeh et al., 2018); VirtualLeaf (Wolff et al., 2019); yal|a (Germann et al., 2019). 